\title{
Editorial
}

\section{Dentistry during COVID-19 Pandemic}

\author{
R. P. Gupta ${ }^{1}$ \\ 1President, International College of Dentist, Section VI (USA), \\ Fellow of Pierre Fauchard Academy, President IDA \\ Chandigarh Branch
}

Dent J Adv Stud 2020;8:35-35

Since the beginning of 2020, Coronavirus disease 2019 (COVID-19) caused by Coronavirus 2 (SARS-CoV-2), first named as the 2019-novel Coronavirus or 2019-NCoV, has rapidly spread throughout the inhabited world and led to unprecedented major health, humanitarian, and financial crises. Efforts to contain the spread of the disease have led to major disruptions, forcing regional and, in many cases, national emergencies and lockdowns, leaving only essential services to continue. Therefore, performing elective tasks as part of any dental treatment have been suspended, as per the orders of the federal state and professional regulatory bodies. Later on, to provide the comprehensive summary of the implications of SARS-CoV-2 and COVID-19 on dental treatment, only contingency management, that is, urgent and emergency care, was allowed after avoiding aerosol-generating procedures.

Dental professionals should check the recommendations made by the federal state or central regulatory bodies if aerosol-producing procedures cannot be avoided.If hand piece is imperative, antiretraction hand pieces are advised to restrict the debris and fluid aspiration and, at a later point in time, ejection. High-volume suction is critical for all aerosol-producing procedures. If radiographs are required, extraoral radiography is preferred over intraoral radiography to reduce saliva and aerosol production due to gagging and coughing. If local anesthesia is required, a local anesthetic gel is preferred over local anesthetic spray due to the potential of virus spread in the air.

Dental procedures must follow rigorous disinfection following the completion of any dental treatment to minimize virus spread through fomites and environmental surfaces. All surfaces should be thoroughly disinfected using hospital-grade disinfectants such as sodium hypochlorite. for coronavirus, bleach/sodium hypochlorite should be used in concentration of 0.1 to $0.2 \%$ for 1 minute. Hydrogen peroxide vaporizer has also been proposed for postprocedural operatory decontamination. Waterlines that have been used should be adequately purified or cleaned to prevent a backflow of pathogens, which can harbor in the plastic tubing. All instruments should be properly disinfected and sterilized immediately (used and unused); disposables that were within the exposed portion of the operatory should be presumed to be infected and disposed of as infected material waste.

The dental clinic must maintain a very high standard of sterilization and infection prevention and control protocol, following the proper sequence of replacement and removal of PPE. The procedural mouth rinse containing $0.2 \%$ Povidine-iodine or $1 \%$ Hydrogen Peroxide is advised. During the pandemic, practitioners should refrain from performing any procedures that lead to aerosol generation, and avoid air water syringes, high-speed handpieces and ultrasonic scalers. The CDC has recommended that N95 masks, eye protection, face shield, gown and gloves should be used for any aerosol-producing procedures. Treatment advice should be provided via telecommunication, that is, remotely first whenever possible and wherever necessary; in person treatment can be performed in a well-prepared operatory, following the recommendations of protocol operatory procedures, guidelines and practice advisories issued by the federal state and local health and regulatory authorities.

The reality is that the future will always remain uncertain. No one predicted the COVID-19 outbreak in late 2019, although Bill Gates famously warned about the potential damage the world would stand to face as a result of a pandemic during a TED TALK in 2015. We acknowledge the uncertain nature of the ongoing COVID-19 pandemic and are growing interconnected and complex worlds to counter the situation. The qualities fundamentally needed are flexibility, robustness, and resilience to deal with unexpected future events and scenarios.

\section{Conflict of Interest}

None declared.
Address for correspondence

R. P. Gupta, BDS(Pb), MDS(PGI), Dental Clinic and Orthodontics

Centre, Chandigarh, India

(email: Rpg817@gmail.com).
DOI https://doi.org/ 10.1055/s-0040-1715548 ISSN 2321-1482.
(C)2020 Bhojia Dental College and Hospital affiliated to Himachal Pradesh University
License terms

() (1) $\ominus \circledast$ 